\title{
Detecting Failure Precursors in BGA Solder Joints
}

\author{
Rubyca Jaai, Graduate Student, University of Maryland \\ Michael Pecht, PhD, University of Maryland \\ Jason Cook, U.S. Army ARDEC
}

Key Words: accelerated testing, anomaly detection, BGA, MSET, SPRT, thermal cycling

\section{SUMMARY \& CONCLUSIONS}

A failure precursor is a measurable parameter which provides an indication of impending failure. By detecting changes in these precursors, deviation of the system from its healthy state can be detected early and unanticipated failures can be avoided. The case study illustrates the use of Multivariate State Estimation Technique (MSET) and Sequential Probability Ratio Test (SPRT) to detect the onset of failure in Ball Grid Arrays (BGA) subjected to Accelerated Temperature Cycling (ATC) tests. The residuals from MSET show a shift in their distribution as they degrade; SPRT detects the anomalous behavior (degradation) in the interconnects. The time to detection of anomalies was found to be earlier than the time to failure of the BGA interconnects, providing a prognostic distance. Hence the change in resistance of the BGA can be used as a precursor to failure.

\section{INTRODUCTION}

Prognostics and health monitoring (PHM) is a method that enables monitoring of the state of reliability of a product in real time, and therefore can be used to provide advance warning of a failure, minimize unscheduled maintenance, provide condition-based maintenance, and help in product design and development [2]. In electronics, the implementation of PHM can be carried out at the product, assembly, or component level to detect and interpret the parameters indicative of performance degradation, physical or electrical degradation, or changes in life cycle environment [1] [2].

Prognostics can be implemented using three approaches: physics-of-failure-based approach, data-driven approach, or by using canaries. The data-driven approaches make health decisions and predictions based purely on the available data collected from the system by continuous monitoring using sensors [4]. In this approach, pattern recognition algorithms are used to detect trends in the data. Environmental and operational loads, as well as system parameters, are monitored and trended based on historical data or known fault states for prediction of component or system failure. Data-driven algorithms combine techniques such as numerical optimization, statistics, and probability to make decisions about current and future system health based on historical data collected from the system [4]. The advantage of using datadriven techniques is that it is not necessary to have product- specific knowledge (i.e., material properties, construction, and failure mechanisms) for its implementation.

The measure of time between the warning from the prognostic system and the actual failure of the system is called the prognostic distance [3]. The parameters monitored for the purpose of anomaly detection are known as precursors. Changes in the monitored parameters as the system degrades are expressed as behavior that is considered anomalous in comparison to the data collected during normal operation of the system. The anomalous behavior leads to subsequent system failure. Therefore, detection of anomalies is used to provide early warning of failure. Precursors are selected to provide prognostic distance. The trend of the precursor data during the healthy state of operation is compared with in-situ data collected from the system for the detection of anomalies. This is done using data-driven algorithms.

In accelerated testing, failures are defined using thresholds, such as increase in resistance to a fixed threshold or a change in capacitance by a certain percentage when subjected to accelerated tests. Failure is detected and failure analysis conducted only when this threshold is exceeded. However, when tests are designed to create fatigue or creepbased degradation, damage accumulates over time. In such cases, the test should be stopped when early signs of degradation are noticed. Not stopping the test could cause changes in failure site morphology, which would make subsequent failure analysis difficult. Analysis of the component before a hard failure, particularly at the time of the earliest detected degradation, can better preserve the failure site for failure analysis. Prognostics can be implemented in accelerated testing to detect early degradation and hence create an opportunity to carry out failure analysis on the component before the failure site morphology is affected by accumulated damage.

The next section describes an approach to detecting degradation in cyclic data using Multivariate State Estimation Technique (MSET) and Sequential Probability Ratio Test (SPRT). This approach has been validated by using data from an accelerated temperature cycling test conducted on BGAs.

\section{APPROACH}

The system is monitored continuously under its life cycle conditions to capture the variation in the parameters for all the operating states. The data from known healthy operating states 
are chosen as training data to represent the system's healthy state. The data obtained has to be processed before being input to the algorithms such that all of the parameters are of the same scale. This is done to avoid the effect of scaling, which could cause errors in the predictions from the algorithms. Scaling effects can cause the estimations from the MSET algorithm to be biased by the parameter(s) at higher scales. The estimations then would not truly reflect the other system parameters as the MSET estimates are calculated using a similarity operator that uses the Euclidean norm function on each vector. The Euclidean norm for a vector $\underline{x}$ having parameters $\mathrm{x}_{1}, \mathrm{x}_{2}$ and so on is calculated using the formula shown in Equation (1).

$$
\|x\|=\sqrt{x_{1}^{2}+x_{2}^{2}+\ldots}
$$

It is therefore required to input normalized data for the estimation of MSET to be more accurate. For cyclic data, the data is rescaled to lie between zero and one. The scaled data is then input to MSET and SPRT for the detection of degradation. Sections 2.1 and 2.2 provide an explanation of these two algorithms.

\subsection{MSET Procedure}

MSET uses pattern recognition to generate an estimate of a system's current health based on healthy data (historic data). The historic data is assumed to cover and provide data for the entire healthy range of the system [6] [7]. The parameters of the system to be monitored are selected and healthy data is chosen for training MSET. The training data is divided into two parts, the memory matrix and the remaining training data. The memory matrix, $\mathrm{D}$, contains the extreme values (highest and lowest) and ordered data chosen at equal intervals from the training set in the form of vectors. The training data that is not used in the memory matrix is known as remaining training data, L. MSET estimates are calculated using a similarity operator on the memory matrix and each newly observed vector. Estimates of the remaining training data, $\mathrm{L}_{\mathrm{est}}$, are obtained and the residuals (the difference between the estimate and observed value) for every observation are calculated. The residuals of the remaining training data represent the healthy state of the system and are called healthy residuals. This represents the training part of the MSET algorithm. Next, estimates $\left(\mathrm{X}_{\mathrm{est}}\right)$ for every new observation from the test data $\left(\mathrm{X}_{\mathrm{obs}}\right)$ are calculated. The operation used to calculate the estimates is the same for estimating both the remaining training data as well as the test data. The test residuals are then calculated. The test residuals describe the deviation of the system under test from its healthy operating condition and are known as the actual residuals. The fault-decision algorithm, SPRT, is then used to compare the actual residuals with the healthy residuals to decide whether the test system is functioning within its normal operating state or not. Figure 1 shows the MSET process.

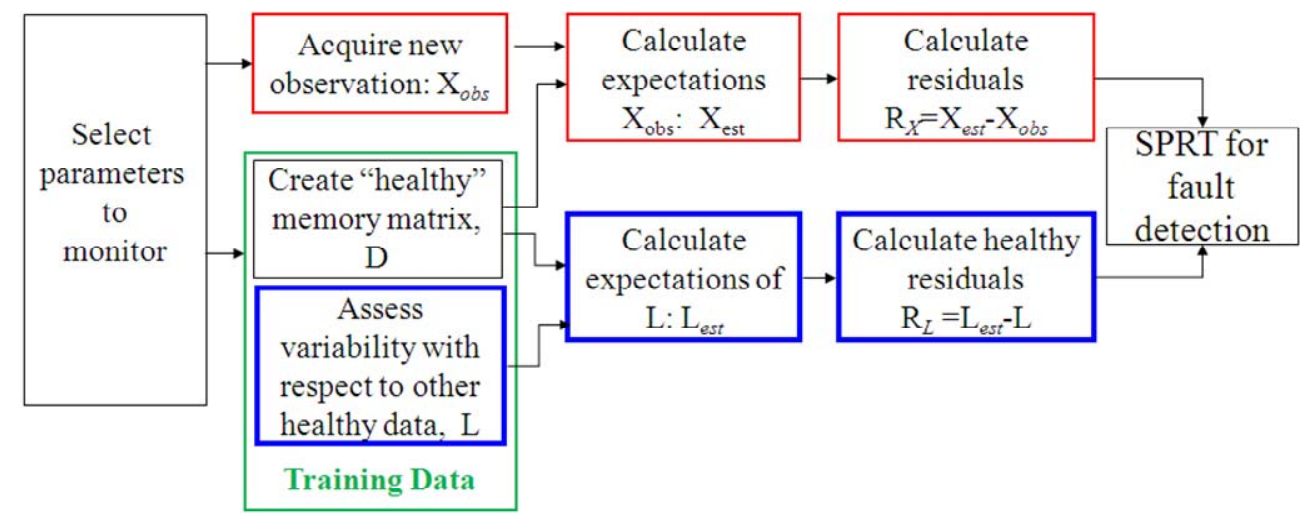

Figure1 - MSET procedure

\subsection{SPRT Procedure}

SPRT is a statistical likelihood ratio test that can be used to make a decision between two or more statistical hypotheses [8] [10] [11]. SPRT is based on the assumption that the data follow the normal distribution. The null hypothesis, $\mathrm{H}_{0}$, represents the healthy state of the system with the data following the Gaussian distribution with mean equal to 0 and standard deviation equal to $\sigma$. Four alternate hypotheses are formulated that represent the degraded state of the system. The hypothesis $\mathrm{H}_{1}$ is when the mean of the data has shifted to $+\mathrm{M}$ with standard deviation $\sigma . \mathrm{H}_{2}$ is when the mean of the data has shifted to $-\mathrm{M}$ with the standard deviation $\sigma . \mathrm{H}_{3}$ and $\mathrm{H}_{4}$ are hypotheses in which the mean remains zero but the standard deviation has increased to $\mathrm{V} \sigma$ and decreased to $V / \sigma$, respectively. The parameters $\mathrm{M}$ and $\mathrm{V}$ are predetermined disturbance magnitudes. These are system-specific and are determined based on system behavior. Figure 2 shows the procedure for SPRT.

SPRT is trained using the healthy residuals from MSET. The mean of the healthy residuals is found and subtracted to get the null hypothesis, $\mathrm{H}_{0}$. The test residual is input and the mean from the healthy residuals is subtracted. Then the SPRT index, which is the natural logarithm of the likelihood ratio, is calculated for each of the alternate hypotheses using Equation (2). 


$$
B \leq \ln \left[\prod_{i=1}^{n} \frac{\operatorname{Pr}\left(x_{i} \mid H_{j}\right)}{\operatorname{Pr}\left(x_{i} \mid H_{0}\right)}\right] \leq A
$$

where

$$
\begin{aligned}
\ln [ & \left.\prod_{i=1}^{n} \frac{\operatorname{Pr}\left(x_{i} \mid H_{j}\right)}{\operatorname{Pr}\left(x_{i} \mid H_{0}\right)}\right] \\
& =\ln \frac{\text { probability of sequence }\left\{X_{n}\right\} \text { given } H_{j} \text { is true }}{\text { probability of sequence }\left\{X_{n}\right\} \text { given } H_{0} \text { is true }} \\
& =\text { SPRT index }
\end{aligned}
$$

$A$ and $B$ are the reject and accept thresholds defined using the false and missed alarm probabilities, $\alpha$ and $\beta$.

$$
A=\ln \frac{1-\beta}{\alpha} \text { and } B=\ln \frac{\beta}{1-\alpha}
$$

If the SPRT index is less than or equal to $B$, then the null hypothesis is accepted and the system is considered to be healthy. The SPRT index is reset and the next test residual is input. If the index is between $A$ and $B$, no decision is made as there is not enough information. In this case the next test residual is input without resetting the SPRT index. If the SPRT index is greater than or equal to $A$, then the alternate hypothesis is accepted. SPRT sets off an alarm and the system is said to be degraded. The SPRT index is again reset and the next test residual is input.

\section{CASE STUDY}

Accelerated Temperature Cycling (ATC) is a well established method for assessing the reliability of test boards. This method is favored due to the fatigue- and creep-induced failures that precipitate on the solder balls of Ball Grid Arrays (BGAs). The boards that were subjected to ATC had polyimide laminates and were populated with eight BGAs thermal expansion (CTE) between the laminate and the package. This causes cyclic shear loading on the interconnects, which leads to degradation and eventually failure. According to IPC-SM-785, failure is defined as the occurrence of a resistance peak $>300 \Omega$ with 9 additional peaks within $10 \%$ of the time to the first peak [12]. Figure 3 shows the behavior of the resistance with change in temperature. It is necessary to remove the effects of the cyclic temperature on the resistance. For this purpose, MSET, which is a non-linear estimation technique, was used to model the lead-lag behavior of the resistance with change in temperature.

The data collected from two BGAs (referred to as BGAs $A$ and B) are analyzed using MSET and SPRT to detect changes in resistance (anomalous behavior). The overall approach is shown in the flowchart in Figure 4.

Resistance and temperature data that represent the healthy behavior of the BGAs are chosen to train MSET. The temperature and resistance are both cyclic in nature, with the temperature ranging from $185^{\circ} \mathrm{C}$ to $-40^{\circ} \mathrm{C}$ while the resistance is observed to be within around $1 \Omega$. Therefore, the two parameters have to be scaled to the same range so that the estimates are not biased or weighted by the changes in temperature alone. The parameters are scaled by dividing each observation by the range (the difference between the maximum and minimum values of the parameter). Then, the minimum value of the parameter is subtracted from every observation. The range and the minimum value are calculated from the training data. The test data vectors are also scaled using the training data range and minimum value. This procedure is carried out for every parameter separately (in this case, for temperature and resistance) and subsequently shifts the values of the parameters to a range from 0 to 1 .

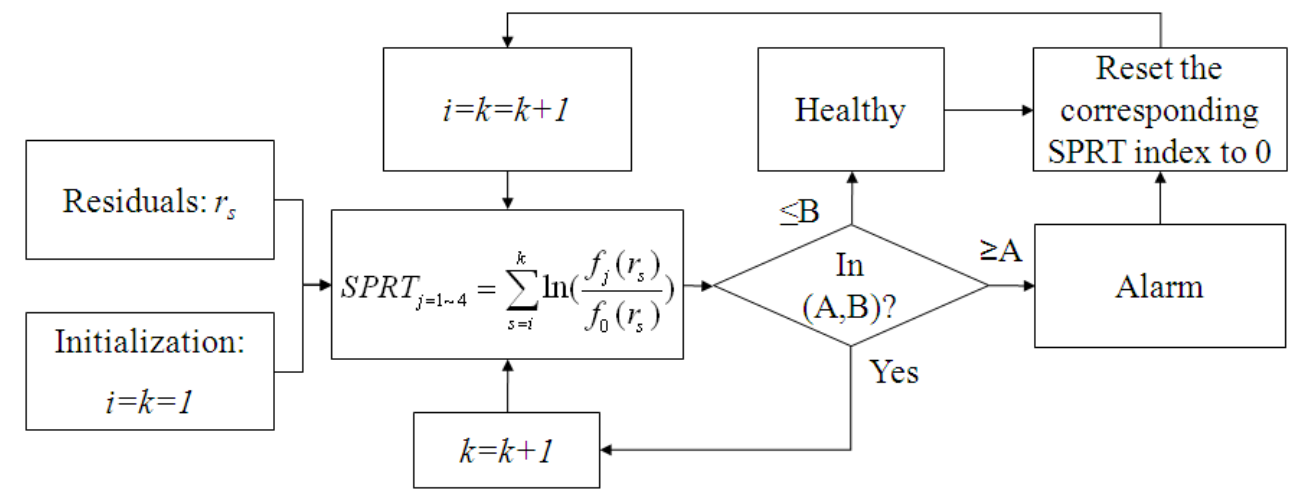

Figure 2 - SPRT procedure

(each having $256 \mathrm{I} / \mathrm{Os}$ ) with separate daisy chains to permit continuous monitoring of resistance. Thermal cycling was performed from $185^{\circ} \mathrm{C}$ to $-40^{\circ} \mathrm{C}$ with dwell times of 15 minutes at both extremes. The ramp rate was chosen to be $3.5^{\circ} \mathrm{C} /$ minute. The temperature and resistance of each BGA was recorded once every minute.

The boards were preconditioned at $100^{\circ} \mathrm{C}$ for $24 \mathrm{hrs}$ as recommended in IPC 9701 [5] before being subjected to the ATC test. During thermal cycling, failure of the BGA interconnects occurs due to the mismatch in the coefficient of
This training data is split into two parts as explained in Section 2.1. The residuals of the remaining training data are used to train SPRT. This is the training phase for both of the algorithms. After training, the procedure is repeated for every test vector from the experiment. The test residuals, obtained for each test vector are then sequentially input to SPRT for anomaly detection. SPRT provides the decision of whether the BGA is showing statistically significant anomalous behavior or is within its healthy operating condition. 


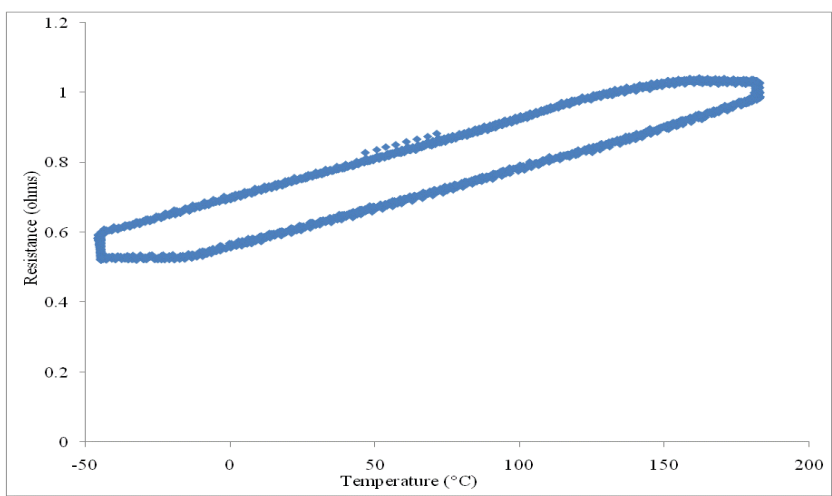

Figure 3 - Resistance vs. temperature

\section{RESULTS AND DISCUSSION}

Two BGAs, A and B, were selected for analysis with four combinations of training and test data shown in Table 1. The alternate hypotheses defined were mean shift value of 0.4 in the residuals of the resistance $(\mathrm{M}=0.4)$. The mean and standard deviation of the healthy residuals are shown in Table 2.

\begin{tabular}{|c|c|c|}
\hline Combination & Training Data & Test Data \\
\hline 1 & BGA-A & BGA-A \\
\hline 2 & BGA-B & BGA-B \\
\hline 3 & BGA-A & BGA-B \\
\hline 4 & BGA-B & BGA-A \\
\hline
\end{tabular}

Table 1 - Combinations of Data for BGA Analysis

Further, the cycle at which alarms were raised by SPRT is tabulated. The results of combinations 3 and 4 are presented in Sections 4.3 and 4.4 to explore the possibility of common training so as to avoid individual training of the algorithm for every component.

\begin{tabular}{|c|c|c|}
\hline Training Data & Mean & Standard Deviation \\
\hline BGA-A & 0.0002 & 0.0017 \\
\hline BGA-B & 2E-05 & 0.0011 \\
\hline
\end{tabular}

Table 1 - Mean and Standard Deviation of Healthy Residuals

\subsection{Combination 1}

Two cycles of resistance and temperature data were chosen from BGA A to train MSET. Test data from BGA A was input to MSET and the test residuals analyzed sequentially using SPRT. SPRT raised the first alarm at cycle 583. Figure 5 shows the residuals of the test data from BGA A and the alarms from SPRT. According to the failure criterion, the BGA failed in cycle 603. It can be seen in the residual data (see Figure 5) that there was an increase in the residual value as the component was subjected to a greater number of temperature cycles, causing a shift in the mean. The

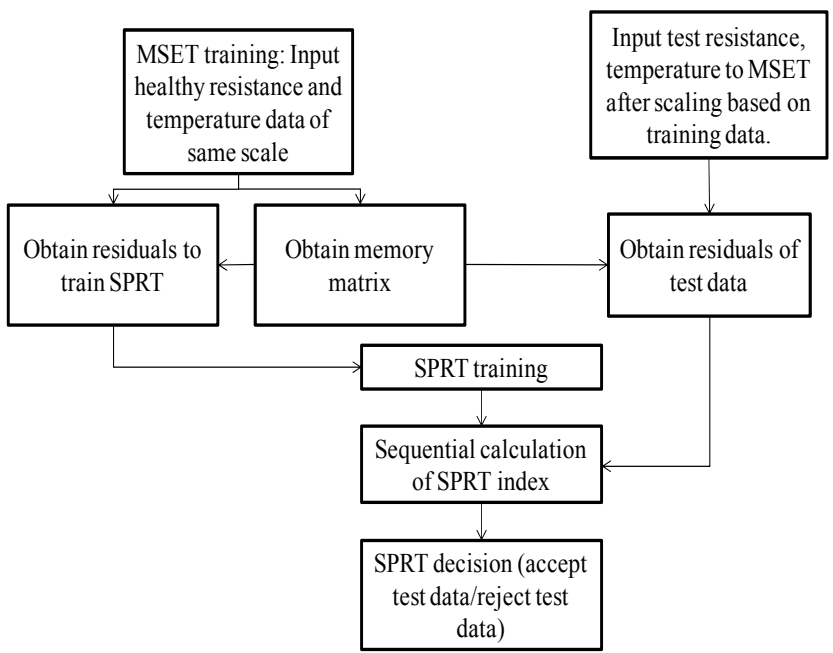

Figure 4-Approach for analysis of BGA data

prognostic distance obtained was equal to 20 cycles

\subsection{Combination 2}

In this case, the training and test data were chosen from BGA B. The first alarm and continuous alarms were seen from cycle 591. According to the failure criterion, the BGA failed in cycle 631 . Therefore, a prognostic distance of 40 cycles was obtained. The value of the prognostic distance varied for each BGA as the change in the resistance in each case was different

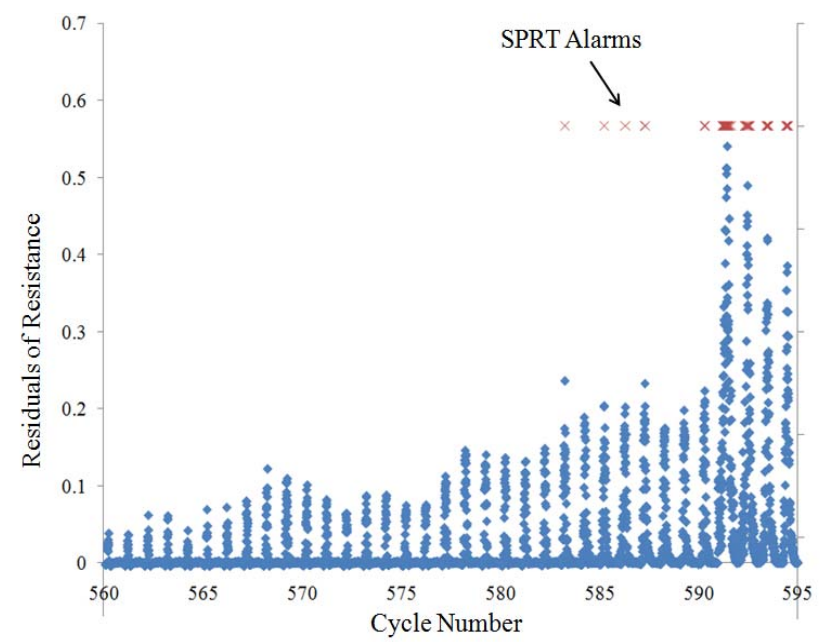

Figure 5 - Test residuals and SPRT alarms for combination 1

near the failure. In the case of BGA $B$, the resistance value increased 40 cycles before the failure criterion was satisfied. Figure 6 shows the test residuals versus the number of cycles and the alarms from SPRT.

\subsection{Combination 3}

Two cycles of training data from BGA A were used (same as that in combination 1). The test data was from BGA B. In this case the SPRT alarms were raised from cycle 405 on. This was due to the resistance of BGA B being greater than BGA A. This caused the MSET estimates to be less accurate since 
the training data (from BGA A) did not include the high

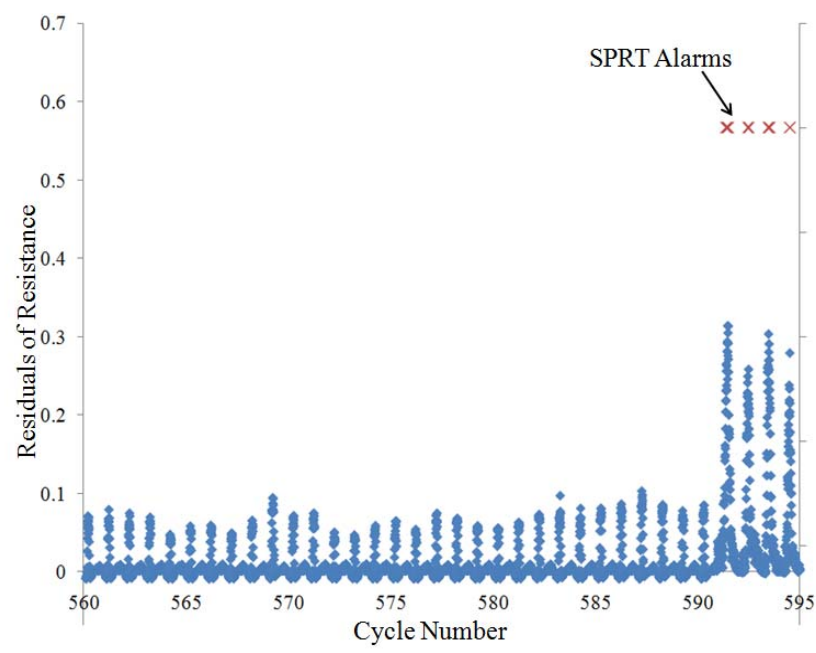

Figure 6 - Test residuals and SPRT alarms for combination 2

resistance values. Therefore, the estimates, particularly at the high temperatures in each cycle, were inaccurate and caused the SPRT to raise false alarms. Further, it was found that the resistance of BGA B drifted during the experiment such that in certain periods the resistance range was similar to component $\mathrm{A}$ and higher in other periods. When the resistance was within the range of BGA A, no alarms were raised. The failure according to the failure criterion was at cycle 631 .

\subsection{Combination 4}

Two cycles of resistance and temperature data were used as training data from BGA B. The training data used was the same as that in Combination 2. Test data in this case was from BGA A. The SPRT alarms were seen from the $600^{\text {th }}$ cycle. According to the failure criterion, failure was in cycle 601. In this case, since the training data was from BGA B, which had higher resistance, accurate estimates were obtained even when the resistance of BGA A increased beyond its normal range. The residuals did not reflect the changes in the component and hence delayed the time of detection of anomalous behavior as compared to combination 4.

\subsection{Discussion}

The use of MSET and SPRT enabled the detection of subtle changes in the data that reflect degradation. The residuals from MSET showed a shift in their distribution as the BGAs degraded. Table 3 shows that the warnings provided significant value in terms of preparing for or avoiding an impending failure when the appropriate training data was chosen, such as in combinations 1 and 2 where the training data was from the same component itself. Combinations 3 and 4 showed that unless the training data chosen was representative of the healthy operating state of the component, the alarms from the algorithms occurred too soon or too late. In the case of the alarms being too early, useful life of the component would be wasted and if the alarms are too late, the failure cannot be prevented.

\begin{tabular}{|l|l|l|l|l|}
\hline $\begin{array}{l}\text { Training } \\
\text { Data }\end{array}$ & Test Data & $\begin{array}{l}\text { SPRT } \\
\text { Alarms } \\
\text { (cycles) }\end{array}$ & $\begin{array}{l}\text { Failure } \\
\text { (cycles) }\end{array}$ & $\begin{array}{l}\text { Prognostic } \\
\text { Distance } \\
\text { (cycles) }\end{array}$ \\
\hline BGA-A & BGA-A & 583 & 603 & 20 \\
\hline BGA-B & BGA-B & 591 & 631 & 40 \\
\hline BGA-A & BGA -B & 405 & 631 & 230 \\
\hline BGA-B & BGA-A & 600 & 601 & 1 \\
\hline
\end{tabular}

Table 2 - Summary of Alarms and Prognostic Distance

\section{FUTURE WORK}

The choice of training data affects the estimations made using MSET. If the training data is not representative of the component, the results from the algorithms will be inaccurate. Therefore, the possibility of using common training for all the BGAs needs to be further studied. A physics-of-failure approach can be incorporated into the analysis for the purpose of providing a remaining useful life prediction. It has been seen that there is the resistance drifts with time, which may be due to causes such as aging. A new approach to training MSET may be necessary to increase the accuracy of the estimates and to better represent the healthy operating condition of the component to prevent the occurrence of false alarms.

\section{REFERENCES}

1. Z. Guangfan, K. Chiman, R. Xu, N. Vichare, and M. Pecht, "An Enhanced Prognostic Model for Intermittent Failures in Digital Electronics," Aerospace Conference, 2007 IEEE, pp.1-8, 3-10 March 2007.

2. M. Pecht, B. Tuchband, N. Vichare, Q. J. Ying, "Prognostics and Health Monitoring of Electronics," Thermal, Mechanical and Multi-Physics Simulation Experiments in Microelectronics and Micro-Systems, EuroSime 2007. International Conference on, pp. 1-8, April 2007.

3. P. Sandborn, “A Decision Support Model for Determining the Applicability of Prognostic Health Management (PHM) Approaches to Electronic Systems," Proceedings of Reliability and Maintainability Symposium, 2005, pp. 422-427, January 2005.

4. M. Pecht, "Prognostics and Health Management of Electronics," 1st Edition, Wiley-Interscience, New York, NY, September 2008.

5. IPC-9701, "Performance Test Methods and Qualification Requirements for Surface Mount Solder Attachments", January 2002.

6. K. Whisnant, K. Gross, and N. Lingurovska, "Proactive Fault Monitoring in Enterprise Servers," Proceedings of the IEEE International Multiconference on Computer Science and Computer Engineering, pp. 3-10, IEEE Press, 2005.

7. N. Zavaljevskl, and K. C. Gross, "Sensor Fault Detection in Nuclear Power Plants Using Multivariate State Estimation Technique and Support Vector Machines," Third International Conference of the Yugoslav Nuclear Society, pp. 1-8. Belgrade, Yugoslavia: Printed in USA 
by Argonne National Laboratory, 2000.

8. A. Wald, "Sequential Analysis," John Wiley \& Sons, New York, NY, 1947.

9. K. C. Gross, and K. Mishra, "Improved Methods for Early Fault Detection in Enterprise Computing Servers Using SAS Tools," Proceedings of SAS® Users Group International Conference 29, Paper 160, pp. 1-9. Montreal, Canada: SAS ${ }$ Users Group International Conference. 2004.

10. K. C. Gross, W. Lu, 2002. Early Detection of Signal and Process Anomalies in Enterprise Computing Systems. Proceedings of the 2002 IEEE International Conference on Machine Learning and Applications, pp. 204-210. Las Vegas, NV: IEEE Press.

11. L. Lopez, "Advanced Electronic Prognostics through System Telemetry and Pattern Recognition Methods," Microelectronics Reliability, pp. 1865-1873, Vol. 47, No. 12, 2007.

12. IPC-SM-785, "Guidelines for Accelerated Reliability Testing of Surface Mount Attachments", November 1992.

\section{BIOGRAPHIES}

Rubyca Jaai,

Center for Advanced Life Cycle Engineering (CALCE)

Department of Mechanical Engineering

University of Maryland

Glenn Martin Hall

College Park, Maryland, 20742 USA

e-mail: rubycajj@calce.umd.edu

Rubyca Jaai is currently pursuing her MS in Mechanical Engineering at the University of Maryland. She earned her bachelor's degree in Mechanical Engineering at the Visvesvaraya Technological University. Her research interests are reliability, prognostics and health management. Her work is focused on diagnostics and prognostics methods for the calculation of remaining useful life incorporating data driven algorithms and physics of failure techniques.

Michael Pecht; PhD

Center for Advanced Life Cycle Engineering (CALCE)

Department of Mechanical Engineering

University of Maryland

Glenn Martin Hall

College Park, Maryland, 20742 USA

e-mail:pecht@calce.umd.edu
Prof Michael Pecht has an MS in Electrical Engineering and an $\mathrm{MS}$ and $\mathrm{PhD}$ in Engineering Mechanics from the University of Wisconsin at Madison. $\mathrm{He}$ is a Professional Engineer, an IEEE Fellow, an ASME Fellow and an IMAPS Fellow. He served as chief editor of the IEEE Transactions on Reliability for eight years and on the advisory board of IEEE Spectrum. He is chief editor for Microelectronics Reliability and an associate editor for the IEEE Transactions on Components and Packaging Technology. He is the founder of CALCE at the University of Maryland, College Park, where he is also a Chair Professor in Mechanical Engineering. He has written more than twenty books on electronic products development, use and supply chain management and over 400 technical articles. He has been leading a research team in the area of prognostics for the past ten years, and has now formed a new Prognostics and Health Management Consortium at the University of Maryland. He has consulted for over 50 major international electronics companies, providing expertise in strategic planning, design, test, prognostics, IP and risk assessment of electronic products and systems. He was awarded the highest reliability honor, the IEEE Reliability Society's Lifetime Achievement Award in 2008. He has previously received the European Micro and Nano-Reliability Award for outstanding contributions to reliability research, 3M Research Award for electronics packaging, and the IMAPS William D. Ashman Memorial Achievement Award for his contributions in electronics reliability analysis.

Jason L. Cook, Ph.D

B62N, Reliability Management Branch, QESA-ARDEC, Picatinny, NJ 07806 USA

e-mail: Jason.cook1@us.army.mil

Jason L. Cook is the Chief of the Reliability Management Branch for the Quality Engineering and System Assurance Directorate of the Armament Research and Development Engineering Center at Picatinny Arsenal, NJ. Jason is responsible for providing consultation, guidance, oversight, and approval of all reliability efforts for the products developed and fielded by ARDEC. In addition, Jason is a member of the American Society of Quality and has met their requirements as a Certified Reliability Engineer (CRE).

Most recently, Jason has also earned a Ph.D. from the School of Systems and Enterprises at Stevens Institute of Technology, where he currently serves as an Adjunct Professor. Dr. Cook's research centers on Network and Distributed System Reliability Analytics. 